\title{
Numerical Model of Cathodic Protection and Its Application in Submarine Pipeline Corrosion
}

\author{
Xu Rongrong, Wang Beifu*, Nie Lihong, Lv Jianxiong, Hao Yongjie, Zhou Jingye \\ School of Petrochemical and Energetic Engineering \\ Zhejiang Ocean University \\ Zhoushan, China, 316000
}

\begin{abstract}
This paper is analyzed the numerical model of cathodic protection, and its application in the damage of submarine pipeline coating. On the basis of the Laplace equation, combined with the boundary conditions required for the damage of the submarine pipeline coating, construct the numerical model that suitable for the damage of the submarine pipeline coating under cathodic protection. In this paper, the cathodic protection potential distribution of the submarine pipeline can be obtained by taking different values of the apparent resistivity of the coating in the boundary condition. The numerical model can be calculated by computer software using the three-dimensional finite element method. And then, analysis the applications of numerical model in the submarine pipeline corrosion combined with its damage rate.
\end{abstract}

Keywords-Cathodic protection, numerical model, coating damage, potential field distribution

\section{INTRODUCTION}

Submarine pipeline is one of the important channels for oil (gas) transportation, and it is also the fastest, most safe and economical way to transport oil (gas) on a long-term large scale. However, the situation of corrosion on submarine pipeline is more severe than the pipeline on the land. Once corrosion leakage occurs, both economic and environmental have to bear huge losses ${ }^{[1]}$.

Therefore, the marine industry production paid more and more protection on the protection of submarine pipe corrosion. Submarine pipeline corrosion control system is usually composed of cathodic protection system and coating, this joint protection method is currently recognized as the most cost-effective measures, cathodic protection technology is also recognized to prevent or reduce the equipment corrosion effective method ${ }^{[2]}$.

At present, the numerical calculation has been widely used in the field of cathodic protection. Thus, the construction of the numerical model as a precursor to the numerical calculation is particularly important in solving the cathodic protection calculation, and it is also an important means to optimize the cathodic protection design. Now the numerical model has been well applied in the cathodic protection of marine and terrestrial oil (gas) pipelines ${ }^{[3]}$. However, the construction of the mathematical model remains to be further studied in terms of the cathodic protection works of the submarine pipeline, especially for the distribution of the cathodic protection potential of the submarine pipeline when the submarine pipeline is corroded. Therefore, it is necessary to study the process of cathodic protection when the submarine pipeline has been corroded, and establish a numerical model which is suitable for the submarine pipeline with cathodic protection. Calculate numerical model, and then obtain the distribution of cathodic protection potential to understand the real tube breakage sea pipelines run. Then the observer will be able to detect the corrosion tendency and take measures to repair the corrosion part, to eliminate security risks, to extend the service life of the sea.

\section{PHYSICAL MODEL OF CATHODIC PROTECTION ELECTRIC FIELD}

The three-dimensional physical model of the submarine pipeline under the cathodic protection is constructed (Fig.1), which provides the basis for the construction of the cathodic protection electric field numerical model.
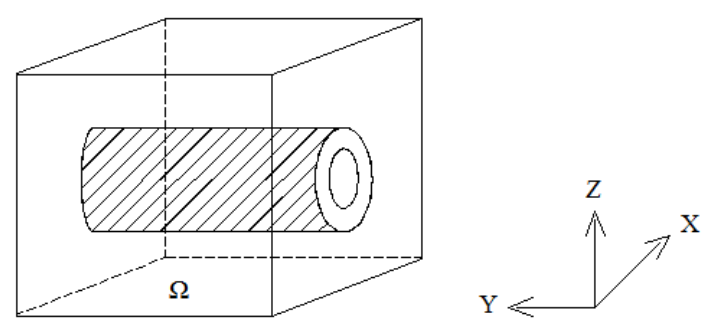

Fig. 1. Three-dimensional physical model of submarine pipeline under cathodic protection

\section{NUMERICAL MODEL OF CATHODIC PROTECTION ELECTRIC FIELD}

\section{A. Selection of mathematical models}

In general, the cathodic protection system numerical model is roughly divided into two kinds - distribution model, time-variant model ${ }^{[4]}$.

Comparing these two numerical models, time-variant model lacks the theoretical basis in the study, and cannot construct the appropriate numerical model to describe the complex and large cyclical change factors in the medium. In the course of the study, the distributed model usually assumes that the system is in a steady state and provides a convenient premise for constructing the potential distribution equation with an electrostatic field. Therefore, this paper chooses the distributed model as the calculation model of the cathodic 
protection numerical model of marine pipeline. When the submarine pipeline is electrochemical corrosion in seawater, the potential distribution in the submarine pipeline and seawater medium conforms to the Laplace equation.

When the cathodic protection system is in the passive electric field of the uniform conductive medium, its potential distribution follows the Laplace equation ${ }^{[5-6]}$ :

$$
\Delta^{2} E=\frac{\partial^{2} V}{\partial^{2} x}+\frac{\partial^{2} V}{\partial^{2} y}+\frac{\partial^{2} V}{\partial^{2} z}=0 \quad((x, y, z) \in \Omega)
$$

Where $\mathrm{E}$ is the cathodic protection potential in region $\Omega$, i is the density of cathodic protection current, and $\mathrm{x}, \mathrm{y}$ and $\mathrm{z}$ are the diagonal coordinate values of the point in the three-dimensional space coordinate system, respectively.

Only by setting the basic boundary conditions can we find the unique solution of the equation (1).

\section{B. Setting up boundary conditions of numerical model}

The boundary condition is a necessary condition for solving the mathematical model of the cathodic protection system. Only the basic boundary condition can be used to set the unique solution of the model. Therefore, the boundary condition has a great influence on the accuracy of the numerical calculation results. It is an important part of the numerical model of the cathodic protection system, and it is also a key problem to solve the numerical model calculation, which is more consistent and more detailed description of the actual situation of the boundary conditions.

Based on the study of corrosion of submarine pipelines under cathodic protection, the distribution of cathodic protection potential field is mainly discussed and the following boundary conditions are proposed:

$$
\Gamma=\Gamma_{0}+\Gamma_{A}+\Gamma_{C}
$$

Where $\Gamma_{A}$ is the auxiliary anode surface; $\Gamma_{C}$ is the outer surface of the pipe where the sea water is in contact with the pipeline, and $\Gamma_{0}$ is the insulation surface. The following is the setting of several boundary conditions.

\section{1) Anodic boundary condition}

Under the premise of cathodic protection, $\Gamma_{A}$ is the boundary of the anode, so the polarization potential of the anode element is $\mathrm{E}$.

In this mathematical model, the anodic potential is taken when cathodic protection is stable:

$$
\left.E\right|_{\Gamma_{A}}=E_{0}
$$

\section{2) Cathode boundary condition}

Cathode boundary conditions, that is, in the vertical cathode surface direction, the relationship between potential and current.

$$
-\left.\mathrm{k} \frac{\partial E}{\partial n}\right|_{\Gamma_{C}}=\frac{1}{R_{P}}\left(E-E_{C}\right)
$$

Where $E_{C}$ is the free corrosion potential of the cathode, $R_{P}$ is the apparent resistivity of the cathode surface, $\mathrm{k}$ is the conductivity of the seawater, and $\mathrm{n}$ is the inner normal direction of the boundary.

For the corrosion of the submarine pipeline studied in this paper, the cathode is basically divided into two different states - the intact area of coating, the damage area of coating: condition:

a) The intact area of coating, Cathode boundary

$$
-\left.\mathrm{k} \frac{\partial \mathrm{E}}{\partial \mathrm{n}}\right|_{\Gamma_{\mathrm{C}_{1}}}=\frac{1}{\mathrm{R}_{\mathrm{P}_{1}}}\left(\mathrm{E}-\mathrm{E}_{\mathrm{C}}\right)
$$

Where $\Gamma_{C_{1}}$ is the intact surface of pipe coating, $R_{P_{1}}$ is the apparent resistivity of the intact coating surface.

b) The damage area of coating, Cathode boundary condition:

$$
-\left.\mathrm{k} \frac{\partial E}{\partial n}\right|_{\Gamma_{C_{2}}}=\frac{1}{R_{P_{2}}}\left(E-E_{C}\right)
$$

Where $\Gamma_{C_{2}}$ is the damage surface of pipe coating, $R_{P_{2}}$ is the apparent resistivity of the intact coating surface.

\section{Insulation surface boundary condition}

The current on the boundary surface is set to constant zero, that is, regardless of its changes over time, the specific definition is as follows:

$$
\left.\frac{\partial E}{\partial n}\right|_{\Gamma_{0}}=0
$$

\section{Establishment of numerical model}

Based on the above considerations and analysis of the mathematical model type and boundary condition setting, the following mathematical model for the cathodic protection potential distribution after submarine pipeline coating is established ${ }^{[7-8]}$ :

$$
\left\{\begin{array}{lr}
\frac{\partial^{2} V}{\partial^{2} x}+\frac{\partial^{2} V}{\partial^{2} y}+\frac{\partial^{2} V}{\partial^{2} z}=0 & ((x, y, z) \in \Omega) \\
\left.E\right|_{\Gamma_{A}}=E_{0} & \left((x, y, z) \in \Gamma_{A}\right) \\
-\left.\mathrm{k} \frac{\partial E}{\partial n}\right|_{\Gamma_{C_{1}}}=\frac{1}{R_{P_{1}}}\left(E-E_{C}\right) & \left((x, y, z) \in \Gamma_{C_{1}}\right) \\
-\left.\mathrm{k} \frac{\partial E}{\partial n}\right|_{\Gamma_{C_{2}}}=\frac{1}{R_{P_{2}}}\left(E-E_{C}\right) & \left((x, y, z) \in \Gamma_{C_{2}}\right) \\
\left.\frac{\partial E}{\partial n}\right|_{\Gamma_{0}}=0 & \left((x, y, z) \in \Gamma_{0}\right)
\end{array}\right.
$$

\section{SURFACE RESISTIVITY OF SUBMARINE PIPELINE}

When the coating of the submarine pipeline is damaged, the actual apparent resistivity $\left(R_{P}\right)$ of the cathode surface is quite parallel to the metal surface resistivity $R_{P}^{\prime}$ and the resistivity of the coating surface $R_{f}{ }^{[9]}$. 


$$
\frac{1}{R_{P}}=\frac{1-f^{\prime}{ }_{c f}}{R_{f}}+\frac{f^{\prime}{ }_{c f}}{R_{P}^{\prime}}
$$

Where, $f^{\prime}{ }_{c f}$ is the surface damage rate.

According to DNV-RP-F103 ${ }^{[10]}$ to get the submarine pipeline coating breakage rate formula:

$$
f^{\prime}{ }_{c f}=\mathrm{a}+\mathrm{b} * \mathrm{t}_{f}
$$

Where, $\mathrm{t}_{f}$ is the service life of submarine pipelines, a and $\mathrm{b}$ are damage constants.

\section{Calculation Method of Numerical Model of CATHODE ELECTRIC FIELD POTENTIAL DISTRIBUTION}

There are three main numerical methods for the commonly used cathodic protection mathematical model: finite difference method (FDM), finite element method (FEM) and boundary element method (BEM).

In this paper, through the numerical model to develop the submarine pipeline corrosion electric field distribution potential calculation software, and then calculated by the pipeline surface potential, in order to obtain pipeline coating situation. Three-dimensional finite element method is more suitable for the two other algorithms because of the complex marine environment, spatial structure and computational workload and precision.

The physical model in Fig. 1 is plotted as shown in Fig. 2 to obtain the finite element geometry model shown in Fig. 3

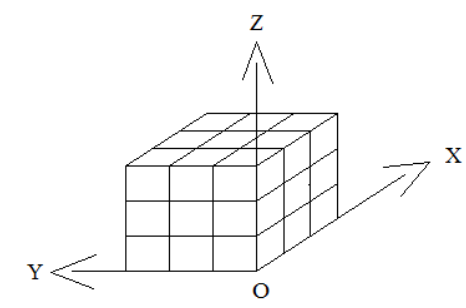

Fig. 2. Stereo element of the finite element model

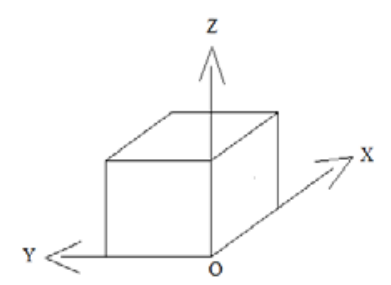

Fig. 3. Geometric model of finite element

The three-dimensional finite element calculation of the cathodic protection numerical model of the submarine pipeline coating is in fact that the $\mathrm{E}, \mathrm{E}_{0}, \mathrm{k}, R_{P_{1}}$ and $R_{P_{2}}$ in the boundary condition are taken into the numerical model according to the actual situation, And then the finite element method is used to solve the problem of the potential distribution $\mathrm{E}$ of the cathode surface.

In order to carry out efficient and accurate calculation of a large number of data, a number of computer software can be used for auxiliary calculation. By comparing the calculated results of the finite element mathematic model with the actual potential data of the sea surface, we can prove the accuracy of the potential distribution result obtained by the mathematical model and the finite element method.

\section{APPLICATION OF NUMERICAL MODEL OF SUBMARINE PIPELINE UNDER CATHODIC PROTECTION}

\section{A. According to the submarine pipeline outside the anti-corrosion coating system and damage analysis of the law to evaluate the cathodic protection effect.}

The equations (4-1), (4-2), and the numerical model (2-8) of the cathodic protection potential distribution are combined to calculate by some calculation softwares. And then, analysis the calculated data, know the different types of pipes in the same period of life under the damage rate and potential distribution, the same pipeline life and damage rate and potential distribution of the relationship, in turn can also predict the situation of the next few years the cathode Protection. So the cathodic protection effect is evaluated according to the breakage rate.

\section{B. Monitoring the damage of the outer anticorrosion layer of submarine pipelines.}

Once the local anticorrosion coating on the submarine pipeline is seriously damaged, the potential curve of the submarine pipeline in the damaged area will obviously fall into "U" shape attenuation. Based on numerical model for single or multiple coating damage location, different damage area of sea pipe anticorrosion layer potential distribution and different damage rate of sea pipe layer potential distribution of external corrosion data are analyzed and compared. In this way, the sea pipe condition can be obtained in time, and timely remedial measures may be taken to avoid losses.

\section{Study the research on the anti - corrosion coating system of the body and joint of the submarine pipeline.}

According to the different types of marine pipe body coating and the sea pipe joint coating, the formula (4-2) set different damage constants for $a, b$. Through the calculation of the model to obtain the respective damage rate, so as to choose the appropriate coating Layer material, optimize the construction program. Under normal circumstances, the damage at the sea pipe joints relative is more serious than other parts of the sea tube, so the joint damage at the potential attenuation is more obvious, leading to the surface of the sea tube cathodic protection potential distribution also presents a different form.

\section{CONCLUSION}

This paper reviews the numerical model of cathodic protection, the numerical model calculation method and the application of the model in the corrosion of submarine pipeline. The numerical model of cathodic protection potential distribution of pipeline is discussed emphatically. The 
numerical model can also be applied to many other areas, such as the evaluation of the cathodic protection effect during the use of the submarine pipeline, the monitoring of the external anticorrosive coating, and the design of the external anticorrosive coating system.

\section{REFERENCES}

[1] Cronin, Duane S., and Pick, Roy J.,Prediction of the failure pressure for complex corrosion defects, International Journal of Pressure Vessels and Piping, 2002, Vol.79 (4), pp.279-287.

[2] Kazuaki Z,Corrosion and life cycle management of portstructures,Elsevier Corrosion Science,2005,47:2353-2360.

[3] Hu Ge,Zhang Shengtao and Xiang Bing,Study on numerical caleulation method in the cathodic Protection of the corrosion field [J] .Computers and Applied Chemistry, 2007,(07):888-890. (In Chinese)

[4] Feng Jiawei,The establishment and application of mathematical mode in cathodic protection of submarine pipeline with damage coating[D], Ocean University of China,2015. (In Chinese)
[5] Sun Wen, Liu Guichang, Wang Lida, Li Yu, A mathematical model for modeling the formation of calcareous deposits on cathodically protected steel in seawater[J], Electrochimica Acta, 2012, Vol.78 , pp.597-608. (In Chinese)

[6] K Zakowski, M Szocinski, M Narozny. Study of the formation of calcareous deposits on cathodically protected steel in Baltic sea water[J]. ANTICORROSION METHODS AND MATERIALS, 2013, 60(2).

[7] Sun Jixing,Wang Qingzhang,Jin Xi,Gao Dayi, Numerical Simulation of Cathodic Protection For Submarine Pipeline on External Anti-Corrosion Coating Breakdown Condition [J]. Total Corrosion Control, 2016,30(11):7-9+85. (In Chinese)

[8] R.E. Melchers, Mathematical modelling of the diffusion controlled phase in marine immersion corrosion of mild steel, Corrosion Science, 2003, Vol.45 (5), pp.923-940.

[9] Bertolini, Luca, Gastaldi, Matteo, Pedeferri, MariaPia, Redaelli, Elena, Prevention of steel corrosion in concrete exposed to seawater with submerged sacrificial anodes, Corrosion Science, 2002, Vol.44 (7), pp.1497-1513.

[10] Tang Jian, Zhang Youhui, Zhang Lin, Ma Yongqing,Cheng Guodong, Analysis on Submarine Pipeline Coating Damage Percentage in the Cathodic Protection Design [J]. Total Corrosion Control, 2015,29(04):57-61. (In Chinese) 